\title{
Investigation of the vibrational properties of cubic yttria-stabilized zirconia: A combined experimental and theoretical study
} \author{
A.P.J. Stampfl ${ }^{\text {b }}$ \\ a School of Physics, The University of Sydney, NSW 2006, Australia \\ b Bragg Institute, Australian Nuclear Science and Technology Organisation, Lucas Heights, NSW 2234, Australia \\ ' Australian Centre for Microscopy E' Microanalysis, The University of Sydney, NSW 2006, Australia \\ ${ }^{\mathrm{d}}$ School of Physics, Monash University, Clayton, Victoria 3800, Australia \\ e Department of Materials Science E Engineering, Yonsei University, Seoul 120-749, Republic of Korea
}

G.P. Cousland ${ }^{\mathrm{a}, \mathrm{b}}$, R.A. Mole ${ }^{\mathrm{b}}$, M.M. Elcombe ${ }^{\mathrm{b}}$, X.Y. Cui ${ }^{\mathrm{a}, \mathrm{c}}$, A.E. Smith ${ }^{\mathrm{d}}$, C.M. Stampfl ${ }^{\mathrm{a}, \mathrm{e}, *}$,

\section{A R T I C L E I N F O}

\section{Article history:}

Received 15 August 2013

Received in revised form 28 October 2013

Accepted 31 October 2013

Available online 8 November 2013

Keywords:

A. Oxides

C. ab initio calculations

C. Neutron scattering

D. Phonons

\begin{abstract}
A B S T R A C T
A combined experimental and theoretical investigation into the vibrational properties of cubic 8-9 mol\% yttria-stabilized zirconia (YSZ) is presented. Measurements of acoustic phonon dispersion curves have been obtained from inelastic neutron scattering investigations using a triple axis spectrometer, as well as calculations of the vibrational density-of-states (vDOS) using density-functional theory. The present measurements agree closely with, and extend, previously published results. The phonons become broader and decrease in intensity as the Brillouin zone boundary is approached, particularly in the $\Gamma-\Delta-X$ direction. Interestingly, there is evidence of a previously unreported low energy phonon band (8-9 meV) in the $\Gamma-\Sigma-X$ direction, which could possibly be related to the stabilization (by yttria doping) of the imaginary mode of cubic $\mathrm{ZrO}_{2}$ about the $X$-point. Compared to pure cubic $\mathrm{ZrO}_{2}$, the vDOS of YSZ are broader and extend to higher frequency. Furthermore, the prominent Zr-related feature in the vDOS of $c-\mathrm{ZrO}_{2}$ at $\approx 14 \mathrm{meV}$ is shifted to higher energy in the vDOS of YSZ. This behavior is consistent with the measured dispersion bands (first acoustic branch in the $\Gamma-X$ direction, about the $X$-point) of YSZ which is higher in energy by a similar amount relative to that of $c-\mathrm{ZrO}_{2}$, thus providing support for the structural model considered.
\end{abstract}

Crown Copyright ๔ 2013 Published by Elsevier Ltd. All rights reserved.

\section{Introduction}

Zirconium oxide $\left(\mathrm{ZrO}_{2}\right)$ or zirconia, has a large range of materials applications because of its high strength and stability at elevated temperatures. Zirconia can be used as a structural ceramic and is an important component in catalytic converters, oxygen sensors, and in chemically passivating surfaces. It is one of the best corrosion-resistant and refractive materials used in metallurgy, and is also utilized as a thermal barrier coating in engines [1-3]. Zirconia is one of the most radiation resistant ceramics currently known, and is of particular interest to the nuclear industry where it has been used, for example, as a passivating medium for hydrogen ingress in pressure tubes [4-6]. The structural and electronic properties of zirconia have been widely reported (e.g. Refs. [7-9]). Zirconia is an insulator, which forms in three temperature dependent polymorphs: Monoclinic zirconia

\footnotetext{
* Corresponding author at: School of Physics, The University of Sydney, NSW 2006, Australia. Tel.: +612935 15901; fax: +61293517726.

E-mail address: stampfl@physics.usyd.edu.au (C.M. Stampfl).
}

$\left(m-\mathrm{ZrO}_{2}\right)$ at ambient conditions [10], tetragonal $\left(t-\mathrm{ZrO}_{2}\right)$ at about $1440 \mathrm{~K}$ [11], and cubic fluorite $\left(c-\mathrm{ZrO}_{2}\right)$ at around $2640 \mathrm{~K}$ [12]. A melt forms at temperatures of about $2950 \mathrm{~K}$.

The addition of yttria $\left(\mathrm{Y}_{2} \mathrm{O}_{3}\right)$ to zirconia results in the ceramic material known as yttria-stabilized zirconia (YSZ) [3]. At ambient temperature and pressure, YSZ can form in a tetragonal phase (for a concentration of 2-9 mol\% yttria) and a cubic phase (4-40 mol\% yttria). Below $4 \mathrm{~mol} \%$, monoclinic $\mathrm{ZrO}_{2}$ is present. A rhombohedral " $\delta$-phase" forms for yttria concentrations above $40 \mathrm{~mol} \%$ [13-15]. The yttrium $(3+)$ cations are aliovalent to the zirconium $(4+)$ cations and this necessitates the inclusion of oxygen vacancies into the lattice, so that charge neutrality is maintained [16]. Yttria stabilized zirconia has applications as ion conductors in solid oxide fuel cells, as oxygen sensors and in various catalysts $[17,18]$. It is also used as thermal barrier coatings (TBC), e.g. for gas turbines [19]. Cubic-stabilized-zirconia has furthermore been proposed as part of the inert fuel matrix in nuclear reactors [20].

The atomic structure of YSZ has been the subject of much past and current research [21-23]. Morell et al. [24] investigated the Raman spectra of cubic YSZ containing $9.5 \mathrm{~mol} \% \mathrm{Y}_{2} \mathrm{O}_{3}$. Spectral analyses of phonon density-of-states indicated results consistent 
with a non-random arrangement of oxygen vacancies. Goff et al. [25] studied YSZ with 9.4-24 mol\% $\mathrm{Y}_{2} \mathrm{O}_{3}$, and reported that vacancies are found in single-vacancy, divacancy or aggregated di-vacancy clusters, depending on the degree of doping. Further, this work proposed that oxygen vacancies occur in vacancyzirconium-vacancy units that are separated along the $\langle 112\rangle$ direction. In a study of cubic YSZ for 9.5-24 mol\% $\mathrm{Y}_{2} \mathrm{O}_{3}$, Argyriou et al. [26], using neutron diffraction, found among other things that the $\mathrm{O}$-atom is predominantly displaced in the $\langle 100\rangle$ direction and to a lesser extent in the $\langle 111\rangle$ direction, and also that appreciable amounts remain at the ideal fluorite sites. From extended X-ray absorption fine structure (EXAFS) studies of the local structure, it was reported that oxygen vacancies are situated as first neighbors to $\mathrm{Zr}$ atoms and $\mathrm{Y}$ atoms are 8-fold coordinated [27].

Theoretically, the atomic structure of YSZ has attracted considerable attention, with progress being made on the basis of firstprinciples density-functional theory (DFT) calculations [28-32]. The results of these theoretical investigations largely support the interpretation of experimental results and show that the structure formed is dependent on the percentage of yttria included [28,29,31-33]. In particular, Bogicevic et al. [28] carried out extensive first-principles investigations and established a number of constraints that the atomic structure of YSZ would follow, related to the stable $\delta$-form of YSZ.

Defects in a crystal structure are well known to potentially induce localized phonon modes, and alter the vibrational densityof-states (vDOS) and the lattice heat capacity. Therefore, studies into the vDOS can give information about the local defect structures (e.g. O vacancies, or Y substituting $\mathrm{Zr}$ ). An understanding of the lattice dynamics is also of interest with regards to the material's ion conduction properties, and also with regard to any technologically important phase transformations. An early work by Liu et al. [34], using inelastic-neutron-scattering, investigated the frequencies and linewidths along the three principle symmetry directions of cubic YSZ with $20 \mathrm{wt} \% \mathrm{Y}_{2} \mathrm{O}_{3}$, for temperatures up to $1700 \mathrm{~K}$. Longitudinal acoustic (LA) and transverse acoustic (TA) phonons were observed, but not optical-phonon branches, consistent with earlier Raman scattering data. This work also reported that acoustic phonons rapidly broaden in the three high-symmetry directions. In a later study by Argyriou and Elcombe [35], the acoustic phonon dispersion curves were measured for three cubic stabilized zirconias (9.5 and $24 \mathrm{~mol} \% \mathrm{Y}_{2} \mathrm{O}_{3}$, and $12.5 \mathrm{~mol} \% \mathrm{CaO}$ ) at room temperature using a triple axis neutron spectrometer. Similarly to Liu et al. [34], LA and TA branches were observed, but optical phonons were not reported.

With regard to theoretical investigations of the vibrational properties and lattice dynamics for $\mathrm{ZrO}_{2}$, such properties have been studied in detail, as well as the various phase transformations (see e.g. Refs. [36-46]). For cubic YSZ, there have only been a few studies, which are briefly described below.

Tojo et al. [47] investigated the thermodynamic properties using molecular dynamics simulations with interatomic potentials, for $8,10,12$ and $14 \mathrm{~mol} \% \mathrm{Y}_{2} \mathrm{O}_{3}$ doped $\mathrm{ZrO}_{2}$. The calculated heat capacity was found to be a maximum at $10 \mathrm{~mol} \%$, which coincides with experimental results. Tojo et al. [47] also calculated the total and partial phonon density-of-states of YSZ for $8 \mathrm{~mol} \% \mathrm{Y}_{2} \mathrm{O}_{3}$. These results showed additional vibrational modes compared to $\mathrm{ZrO}_{2}$ at low frequencies, caused by structural defects in the crystal. In another theoretical study, Shelling et al. [48] performed classical molecular-dynamics simulations of the temperature dependence of the thermal conductivity of $\mathrm{ZrO}_{2}$ and YSZ, for a range of concentrations. They also reported the density of local frequencies for 4 and $20 \mathrm{~mol} \%$ yttria YSZ, finding that the disorder in the oxygen local frequencies is increased, but the range of the distributions is largely unchanged. Ostanin et al. [16,49] performed first-principles theoretical investigations of YSZ between 3 and
$10 \mathrm{~mol} \% \mathrm{Y}_{2} \mathrm{O}_{3}$ using density-functional theory (DFT) and the pseudopotential approach. They calculated the vibrational mode corresponding to the zone-boundary soft phonon, $X_{2}^{-}$, of cubic $\mathrm{ZrO}_{2}$, for YSZ between 3 and $10 \mathrm{~mol} \% \mathrm{Y}_{2} \mathrm{O}_{3}$, where the results showed a phase transition to cubic YSZ at $10 \mathrm{~mol} \% \mathrm{Y}_{2} \mathrm{O}_{3}$.

Lau and Dunlap [50] used DFT calculations to investigate the dielectric, thermodynamic and phonon properties of YSZ structures, predicted from previous calculations of ground state struc-

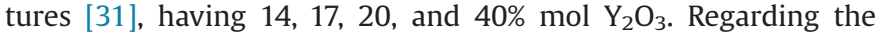
phonons studied, they report the total phonon density-of-states for the so-called $\delta$-phase of YSZ for $40 \mathrm{~mol} \% \mathrm{Y}_{2} \mathrm{O}_{3}$, but not for lower concentrations. In the work of Dalach et al. [32], using a cluster expansion and a statistical thermodynamics approach based on DFT results, among other findings, they predicted the atomic ordering as a function of dopant concentration, where it was found that below $9 \mathrm{~mol} \% \mathrm{Y}_{2} \mathrm{O}_{3}$, cation dopants show a tendency to aggregate. Regarding total phonon density-of-states, results were presented for 33 and $66 \mathrm{~mol} \% \mathrm{Y}_{2} \mathrm{O}_{3}$, in which it was shown that cations are primarily responsible for low-frequency modes at 5-6 THz (21-25 meV), mixed cation-anion participation for 7-15 THz (29-62 meV), and high frequency modes primarily resulting from anion vibrations at $15-23 \mathrm{THz}(62-95 \mathrm{meV})$

In the present paper we perform inelastic neutron scattering experiments for $\mathrm{YSZ}$ with $8-9 \mathrm{~mol} \% \mathrm{Y}_{2} \mathrm{O}_{3}$ and discuss the results in relation to phonon density-of-states calculated using DFT, for two model atomic structures based on predictions from extensive $a b$ initio investigations of ground state structures [28]. One of these structures contains a long-range structural motif (O-vacancy-ZrO-vacancy) oriented along the $\langle 112\rangle$ direction, a configuration considered based on the findings of Goff et al. [25]. This structural model has also been used in first-principles calculations in which the Kohn-Sham eigenvalues are compared with core-level binding energies measured by X-ray photoelectron spectroscopy (XPS) [51]. To the best of our knowledge, calculated first-principles total and partial phonon density-of-states for this concentration of YSZ and comparison with experiment, have not been presented to date. The paper is organized as follows: In Section 2, the experimental procedure and results are described, followed in Section 3 by the theoretical method and results. Section 4 contains the conclusions.

\section{Experiment}

The Taipan thermal triple-axis spectrometer (TAS) [53] at the OPAL reactor [52] located in Sydney, Australia, was used to map phonon dispersion for $\mathrm{YSZ}(100)$ with $8-9 \mathrm{~mol} \% \mathrm{Y}_{2} \mathrm{O}_{3}$. TAIPAN is similar in concept to triple-axis spectrometers IN8 [at the Institut Laue-Langevin (ILL)] and PUMA [Research Neutron Source, Heinz Maier-Leibnitz (FRM-II)] that allow variable incident and final energies, and have a secondary spectrometer with a single detector [54]. The location of TAIPAN is on a tangential thermal neutron beam tube at the OPAL reactor shielded face. For these experiments TAIPAN uses the (002) planes of a highly oriented pyrolitic graphite (HOPG) as the double-focusing monochromator and analyzer. For TAIPAN, the secondary spectrometer consists of separate sample, analyzer and detector stages, which are mounted on air pads that move on a polished granite floor. The secondary spectrometer is conventional and has polyethylene shielding installed around the analyzer and detector elements. The incident energy range of TAIPAN TAS is from $\approx 5 \mathrm{meV}$ to $\approx 80 \mathrm{meV}$. There is a monitor positioned downstream from the monochromater that counts the number of neutrons in the incident beam and is used as a gate to dose the same number of neutrons incident on the sample for each $E_{n}(\mathrm{Q})$ point measured. Instrument control and 
data acquisition were carried out using SpICE, a program [54] written in LabView.

Constant-Q scans, were in this case conducted, over a range of energy transfer, $e_{n}$, by scanning the incident energy, $e_{i}$, at $e_{f}=14.87 \mathrm{meV}$, for a selected point in reciprocal (Q-) space. For constant-energy (constant-E) scans, energy transfer is kept constant, while a line through Q-space is scanned by scanning either the incident or outgoing neutron beam, or by scanning both. The sample was aligned to the beam and the spectrometer and measurement plane defined by using two crystallographic axes, the [200] and [020]. Transverse acoustic (TA) and longitudinal acoustic (LA) phonons were measured by constant-Q and constant-E scans depending on whether the phonon curve was flat or steeply dispersing in energy, respectively. Phonon peak positions were determined by fitting with a Gaussian function. Error bars were calculated assuming Poisson statistics, where the error is the square root of the count. Phonon dispersion curves were measured along the $\Gamma-\Delta-X$ and $\Gamma-\Sigma-X$ directions.

The two YSZ (100) single crystals analyzed each have a concentration between 8 and $9 \mathrm{~mol} \% \mathrm{Y}_{2} \mathrm{O}_{3}$ and are supplied by MaTecK $(\mathrm{GmbH})$. The crystal dimensions are $10 \times 10 \times 1 \mathrm{~mm}^{3}$ (for "Experiment 1") and $10 \times 10 \times 5 \mathrm{~mm}^{3}$ (for "Experiment 2"). Before commencement of these experiments the crystals are characterized with X-ray diffraction (XRD) in a Bragg-Brentano geometry. The analysis shows intense peaks due to 200 and 400 reflections, indicative of a (100) surface cut crystal, and no impurities. Phonon measurements are taken which are compared with previous results of Argyriou and Elcombe [35], who used a YSZ crystal with a concentration of $9.5 \mathrm{~mol} \% \mathrm{Y}_{2} \mathrm{O}_{3}$. The crystal used in the work of Ref. [35] was uncut, with dimensions of approximately $10 \times 10 \times 10 \mathrm{~mm}^{3}$. The volume of the crystal used in "Experiment 1 " is thus 0.1 times that of the crystal used by Ref. [35], but due to the improved neutron flux at Taipan TAS, measurements of the smaller crystal can be compared with published results.

In Experiment 1, TA and LA phonons are mapped in both the $\Gamma-\Delta-X$ and $\Gamma-\Sigma-X$ directions of the Brillouin zone. Phonon dispersion curves are successfully obtained for energy transfer values of $18 \mathrm{meV}$ and less. In "Experiment 2", TA phonons are mapped in the $\Gamma-\Sigma-X$ direction and energy transfer values are measured up to $34 \mathrm{meV}$. In order to have similar signal-to-noise ratios, the duration of Experiment 2 point measurements are typically less than those of Experiment 1, because the larger crystal used in Experiment 2 scatters more neutrons per unit time. All measurements are conducted at room temperature.

For Experiment 1, in order to find the TA phonons along the $\Gamma-\Delta-X$ direction, constant Q-scans were run at $\mathbf{k}$-points $(2.0,2 . x$, $0.0)$, for $x=2,3,4,5$, and 75 and at the $X(2,3,0)$ point. At the $X$ point the lowest energy is measured to be $18.31 \mathrm{meV}$, and is comparable with the result of Argyriou and Elcombe [35] of $19.81 \mathrm{meV}$. In Experiment 1, constant-Q scans are also mapped in the $X-\Sigma-\Gamma$ direction in $\mathbf{k}$-space. Scans are performed at $\mathbf{k}$ points $(1.8,2.2,0.0),(1.6,2.4,0.0)$ and $(1.25,2.75,0.0)$. In Experiment 1, LA phonons are mapped along the $\Gamma-\Delta-X$ and $\Gamma$ $\Sigma-X$ directions. Constant-E scans are acquired from the (220) $\Gamma$ point for energy transfer values of 8 and $16 \mathrm{meV}$. For Experiment 2 , constant-Q scans are performed at $(x, x, 0.0), x=2.4,2.45$, and 2.5 , and over an energy transfer range of $6-34 \mathrm{meV}$.

\subsection{Results: phonon dispersion curves}

Constant-Q scans along the $\Gamma-\Delta-X$ direction are shown in Fig. 1. The phonon branch disperses in energy as expected along most of this symmetry direction, with higher energies on approach to the $X$-point compared to calculations for pure $\mathrm{ZrO}_{2}$. The same TA phonon branch is also measured as constant-Q scans along the $X-\Sigma-\Gamma$ direction. As in Fig. 1, these peaks also broaden

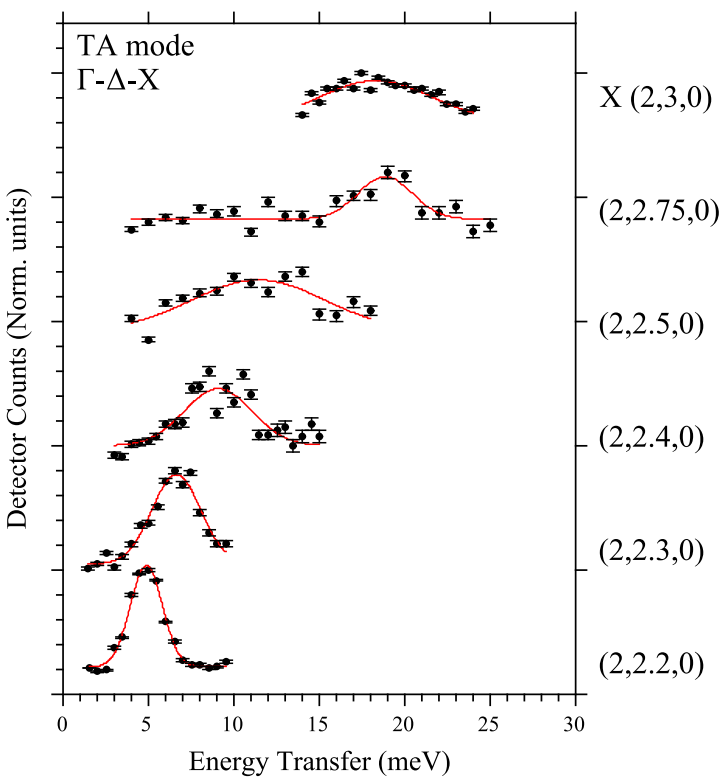

Fig. 1. Constant-Q scans of the TA mode along the $\Gamma-\Delta-X$ direction. Gaussian fits in red. (For interpretation of the references to color in this figure caption, the reader is referred to the web version of this article.)

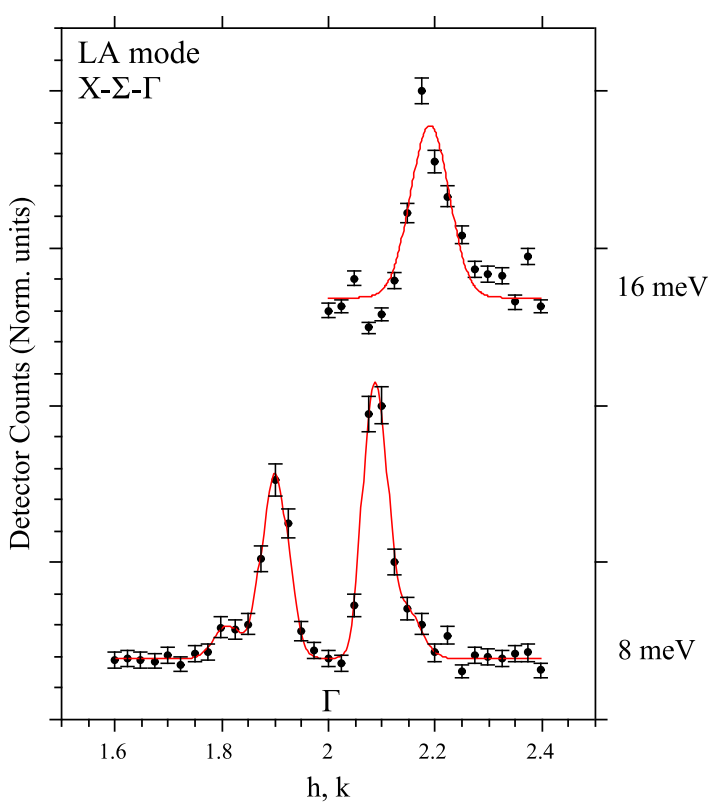

Fig. 2. Constant-E scans for the LA mode along the $\Gamma-\Sigma-X$ direction. Gaussian fits in red. (For interpretation of the references to color in this figure caption, the reader is referred to the web version of this article.)

in energy for higher energy-transfer values towards the $X$-point. Interestingly, phonon scattering is observed at low energies and $Q$-values corresponding to a low energy TA-branch not predicted for this region of reciprocal space [34], that appears to correspond to a complex mode of $\mathrm{ZrO}_{2}$ [44]. This observation is discussed in Section 3.

Constant-E scans along the $X-\Sigma-\Gamma$ direction are shown in Fig. 2. The energy transfer values of these scans are $8 \mathrm{meV}$ (lower plot) and $16 \mathrm{meV}$ (upper plot). This branch follows quite well the calculation and previous results [34]. Constant-E scans for an energy transfer of $8 \mathrm{meV}$ are also measured along the $\Gamma-\Delta-X$ direction (not shown). Both TA and LA low energy branches near the $\Gamma$-point are observable and correspond well to previous measurements and calculation [34]. 
Similar to Argyriou and Elcombe [35], optical phonon modes were not observed.

In Fig. 3a and b, the two-dimensional extended Brillouin zone and measurement plane is shown, where the Q-space position of each of the scans is mapped. The Q-space positions of the TA phonon branch are indicated by green dots and the LA branch by blue dots. The green dots represent the positions in the Brillouin zone at which the constant-Q scans were measured. The blue dots represent the Q-space LA peak positions where constant-E scans were measured.

Fig. 4 shows the results of both Experiments 1 and 2, where those of Argyriou and Elcombe [35] are also plotted. Overlaid is the calculated result, obtained using a self-consistent $a b$ initio lattice dynamical method (with a 192-atom supercell), for cubic $\mathrm{ZrO}_{2}$ of Souvatzis and Rudin [44] for high-symmetry lines of the Brillouin zone. The result shows the well-known soft phonon mode at the $X$-point, indicating the instability of the cubic phase. (We note that Born effective charges were not included in this calculation, therefore, longitudinal optical/transverse optical splitting at the $\Gamma$-point was not obtained.)

From Fig. 4, the present results for TA and LA phonons in the $\Gamma-\Delta-X$ region are close to those of Argyriou and Elcombe [35]. For example, the position of the $X_{1}$-point obtained in the present (Experiment 1) result is $18.3 \mathrm{meV}$ and that of Argyriou and Elcombe [35] is $19.8 \mathrm{meV}$. This is so even though the crystals are of slightly different $\mathrm{Y}_{2} \mathrm{O}_{3}$ concentrations (8-9 mol\% $\mathrm{Y}_{2} \mathrm{O}_{3}$ and $9.5 \mathrm{~mol} \% \mathrm{Y}_{2} \mathrm{O}_{3}$, respectively). In the $\Gamma-\Sigma-X$ region, the $\Sigma$ (LA) phonons measured in Experiment 1 (blue triangles in Fig. 4) are also close to the result of Argyriou and Elcombe [35], and to the earlier work of Liu et al. [34]. In Fig. 4 the calculated phonon bands of Liu et al., obtained by the rigid-ion model calculated using a best-fit to the measured acoustic branches of Ref. [34], are also overlaid.

It is interesting to note that the $\Sigma$ (LA) dispersion band, mapped from the results of Experiments 1 and 2 (blue triangles and downward pointing purple triangles in Fig. 4) correspond well to the $\Sigma$ (LA) phonon band measured by Argyriou and Elcombe (red rectangles in Fig. 4) [35]. This dispersion band is expected, from the theory, to meet with the other bands (in the $\Gamma-\Delta-X$ region) at the $X$-point. In order to confirm this, further measurements of $\Sigma$ (LA) phonons would be required close to the $X$-boundary of the $\Gamma-\Sigma-X$ region. Interestingly, evidence for another $\Sigma(\mathrm{LA})$ dispersion band in the $\Gamma-\Sigma-X$ region was obtained (see three peaks around $8-9 \mathrm{meV}$ in Fig. 4). The origin of these features is unclear, but they could possibly be related to the soft longitudinal mode that is imaginary for pure $c-\mathrm{ZrO}_{2}$ (see pale dashed line in Fig. 4), which on doping with yttria, moves upwards and becomes positive. The work of Souvatzis and Rudin shows that for simulations of the phonon curves of $c-\mathrm{ZrO}_{2}$ at high temperature $(2570 \mathrm{~K})$ (see Fig. 2 of Ref. [44]), this band increases in energy, becoming positive as $c-\mathrm{ZrO}_{2}$ becomes stable. Further measurements would be required to map the dispersion across as much of the $\Gamma-\Sigma-X$ line as possible in order to confirm this.

Compared to the phonon dispersion curves of $c-\mathrm{ZrO}_{2}$, a noticeable difference to the measured curves of YSZ is that the position of the lowest energy TA branch at the X-point is higher in energy

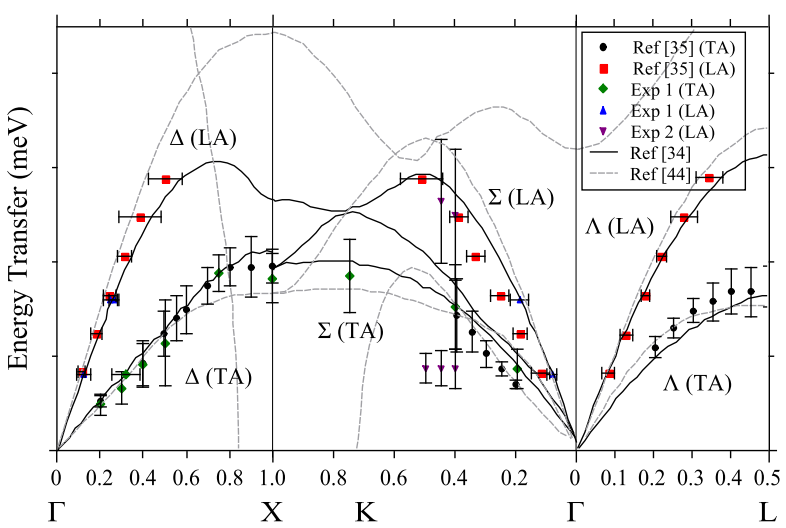

Fig. 4. Phonon dispersion results obtained in the present work (denoted by "Exp 1" and "Exp 2"), and those of Argyriou and Elcome [35]. Theoretical dispersion curves obtained from first-principles calculations for pure $\mathrm{ZrO}_{2}$ (grey dashed lines) of Ref [44], and the dispersion curves for YSZ obtained by the rigid-ion model and fitting to the experimental acoustic branches (black lines) [34].
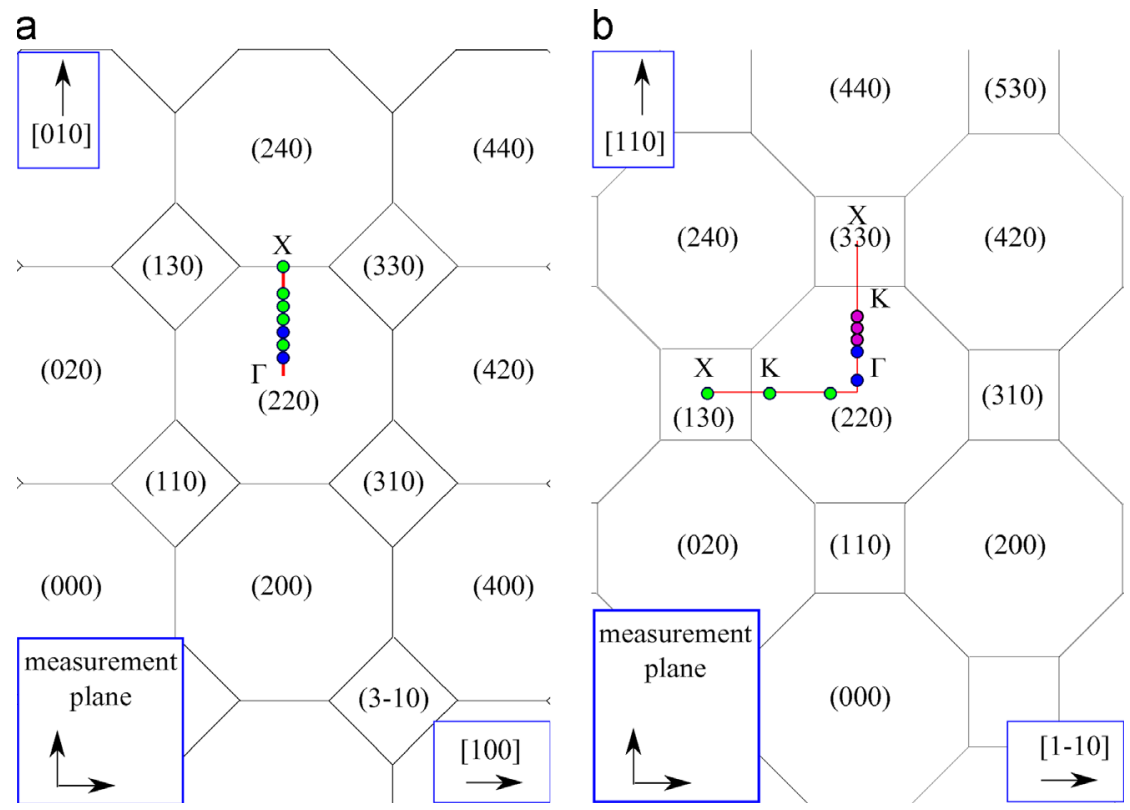

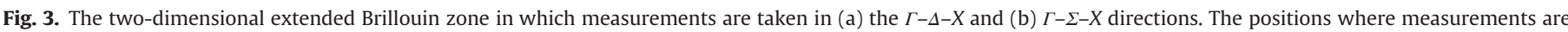

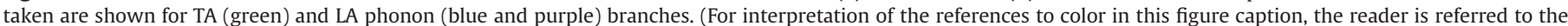
web version of this article.) 
Table 1

The peak positions of the vDOS for cubic $\mathrm{ZrO}_{2}$ compared with analogous results obtained from Kuwabara et al. [43] and Sternik and Parlinski [42]. The values of Refs. $[42,43]$ are obtained from the published vDOS figures.

\begin{tabular}{llll}
\hline Peak label & Present, meV (THz) & $\begin{array}{l}\text { Kuwabara et al. [43] } \\
\text { meV (THz) }\end{array}$ & $\begin{array}{l}\text { Sternik et al. [42] } \\
\text { meV (THzs) }\end{array}$ \\
\hline A (Zr) & $8(2)$ & - & - \\
B (Zr) & $14(3.4)$ & $12(3)$ & $17(4)$ \\
C (Zr) & $28(6.8)$ & $27(6.5)$ & $33(8)$ \\
D (O) & $27(6.5)$ & $27(6.5)$ & $33(8)$ \\
E (O) & $44(10.6)$ & $43(10.5)$ & $48(11.5)$ \\
F (O) & $54(13)$ & $52(12.5)$ & $58(14)$ \\
G (O) & $60(14.5)$ & $60(14.5)$ & $66(16)$ \\
H (O) & $67(16.2)$ & $70(17)$ & $70(17)$ \\
I (O) & $76(18.4)$ & $77(18.5)$ & $83(20)$ \\
\hline
\end{tabular}

Table 2

Experimental results of high symmetry points in the phonon dispersion curve (see Fig. 4) for YSZ at $9.5 \mathrm{~mol} \% \mathrm{Y}_{2} \mathrm{O}_{3}$ [35] and 8-9 mol\% $\mathrm{Y}_{2} \mathrm{O}_{3}$ (present work). The energy transfer values of these points are correlated with frequency values of peaks in the calculated vDOS (for the structure shown in Fig. 5), which are also listed.

\begin{tabular}{|c|c|c|c|c|}
\hline Curve & $\begin{array}{l}\text { Exp 1, Exp } \\
2 \text { - Energy transfer } \\
(\mathrm{meV})\end{array}$ & $\begin{array}{l}\text { Reference [35] } \\
\text { - Energy transfer } \\
(\mathrm{meV})\end{array}$ & $\begin{array}{l}\text { vDOS } \\
\text { peak } \\
\text { label }\end{array}$ & $\begin{array}{l}\text { vDOS } \\
\text { value } \\
\text { (meV) }\end{array}$ \\
\hline$X$-point & 18.3 & 19.8 & $1(\mathrm{Zr})$ & 17.3 \\
\hline$L$-point & & $\sim 17.4$ & $2(\mathrm{Y})$ & 15.8 \\
\hline Exp 2 & 8.7 & & $3(\mathrm{Zr})$ & 6.3 \\
\hline
\end{tabular}

in the latter material (by $4-5 \mathrm{meV}$ ). In Table 2 the experimental results at high symmetry points in the phonon dispersion curve (Fig. 4) are collected, where they are correlated with frequency values of peaks in the calculated vDOS (described below).

\section{Calculation method and phonon density-of-states}

The all-electron density-functional theory (DFT) calculations are performed using the $\mathrm{DMol}^{3}$ code [55], with the generalized gradient approximation (GGA) [56] for the exchange-correlation functional. The wave functions are expanded in terms of a doublenumeric quality localized basis-set with polarization functions (DNP) and an atomic real-space cut-off of 9 Bohr. For calculating the properties of bulk cubic $\mathrm{ZrO}_{2}$, the Brillouin-zone integrations are performed using a $(4 \times 4 \times 4)$ Monkhorst-Pack (MP) grid. These parameters are selected after performing convergence tests described in Ref. [57]. The optimized lattice constant is $5.14 \AA$, which is in good agreement with that obtained by Bogicevic et al. [28] of $5.16 \AA$, and close to the experimental value of $5.09 \AA$ (obtained by an extrapolation of the high-pressure value to zero temperature [58]).

For calculation of the phonon (vibrational) density-of-states (vDOS) of pure $\mathrm{ZrO}_{2}$, converged results are obtained using a large 216 atom supercell, corresponding to the 12-atom conventional cell being repeated in the $x-, y$-, and $z$-directions 3,3 , and 2 times, respectively (details in Ref. [57]). For the k-point mesh of the 216atom supercell, a $(2 \times 2 \times 2)$ grid is used. To describe the YSZ material, two structural models are considered, both corresponding to $\sim 10 \mathrm{~mol} \%$ yttria. The first is constructed based on the 12 atom fluorite $\mathrm{ZrO}_{2}$ conventional cell, doubled in each cardinal direction, resulting in a 96-atom supercell. In this supercell, six yttrium atoms are substituted for zirconium atoms and three oxygen atoms are removed (resulting in a 93-atom supercell). The atomic structure employs a constraint, based on results from neutron and X-ray scattering experiments, that oxygen vacancies align along the $\langle 111\rangle$ direction [25]. A further constraint is that yttrium atoms are located at next-next-nearest neighbour positions to oxygen vacancies [29].

The second structure (shown in Fig. 5), includes extra constraints proposed in the work of Goff et al. [25]. In this model the oxygen vacancies are included only in vacancy-zirconium-vacancy motifs that are separated along the $\langle 112\rangle$ direction of the YSZ supercell. This model also includes the constraints used in the first structural model. The resulting supercell is twice that of the previous model (i.e. contains 186 atoms). To achieve convergence of the vDOS for the first (93-atom supercell) model, it was necessary to double, the supercell [57]. For all these calculations, the internal atomic positions were fully relaxed. A $(2 \times 1 \times 2)$ k-point set was used for the second structure (i.e. the same sampling as for the 93-atom supercell). It was found that the second model is energetically more favorable than the first, and the calculated vDOS of both structures is very similar, therefore only the vDOS of the second model is presented and discussed in the paper.

\subsection{Results: vibrational density-of-states}

In Fig. 6 the total and partial vibrational density-of-states (vDOS) of $\mathrm{ZrO}_{2}$ are shown (dashed curve). Between $25 \mathrm{meV}$ and $50 \mathrm{meV}$ there are contributions from coupled $\mathrm{O}-\mathrm{Zr}$ vibrations,

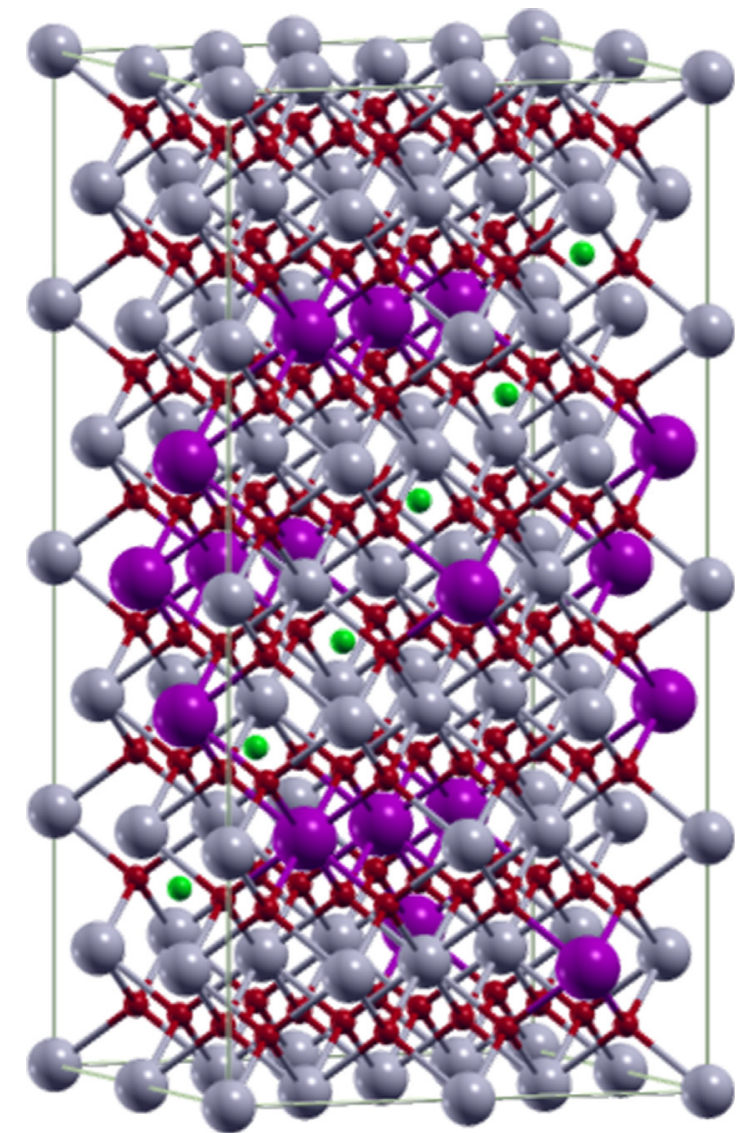

Fig. 5. Atomic structure of model created using a 186-atom supercell of YSZ ( $\sim 10 \mathrm{~mol} \%$ yttria). The zirconium atoms are shown as large grey spheres, the yttrium atoms as large dark (purple) spheres, oxygen atoms as small dark (red) spheres, and the position of the oxygen vacancies as small unbonded grey (green) spheres. The oxygen vacancies are located along the $\langle 111\rangle$ direction and form O-vacancy-Zr-O-vacancy units that are separated along the $\langle 112\rangle$ direction. The yttrium atoms are positioned as next-next nearest neighbours to the oxygen vacancies. (For interpretation of the references to color in this figure caption, the reader is referred to the web version of this article.) 


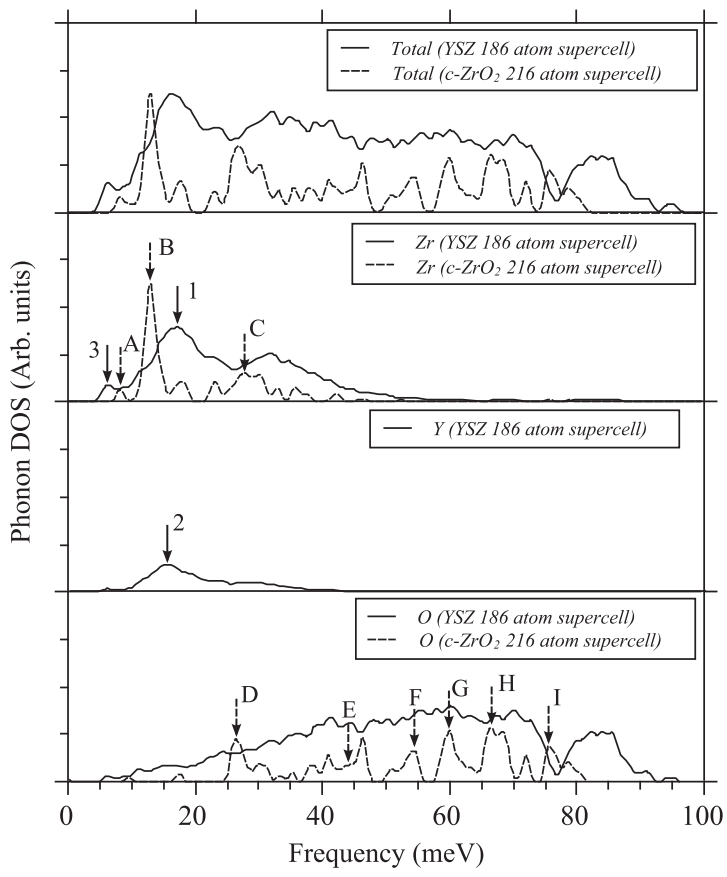

Fig. 6. Comparison of total and partial vDOS for cubic $\mathrm{ZrO}_{2}$ (216 atom supercell) and YSZ (186 atom supercell) with $\sim 10 \mathrm{~mol} \% \mathrm{Y}_{2} \mathrm{O}_{3}$.

while for frequencies $>50 \mathrm{meV}$, the main contribution is due to the $\mathrm{O}$ atoms. For energies lower than about $25 \mathrm{meV}$, contributions from the cations are dominant. The peaks in the vDOS correspond to regions in the phonon dispersion curves with lowest gradient, typically close to the Brillouin zone boundaries. The calculated vDOS are in good agreement with those of previous ab initio calculations (e.g. Refs. [42,43]). This is demonstrated by the comparison in Table 1 of the peak positions (indicated with arrows in Fig. 6) obtained from the present results, and those from Kuwabara et al. [43] and Sternik and Parlinski [42]. Similar to Kuwabura et al. [43] the present calculations do not include Born effective charges and thus the longitudinal optical/transverse optical splitting at the $\Gamma$-point is also not described. The result of Sternik and Parlinski, however does describe this splitting, which does not appear to alter very much the shape of the total vDOS (seen from inspection of Figs. 1a and 4, in Refs. [43,42], respectively). It is interesting to note that there is a prominent O-related vDOS peak in Kuwabara et al. and Sternik and Parlinski at $70 \mathrm{meV}$ that is considerably smaller in the present work (labeled $\mathrm{H}$ in Fig. 6). A similarly smaller feature was obtained in the recent study by Souvatzis and Rudin [44] using the largest (192-atom) supercell considered in that work. This peak was notably larger in their results when calculated using smaller (24and 81-atom) supercells, suggesting that this peak is sensitive to the calculation details (i.e. system size). A small peak due to $\mathrm{Zr}$ can be seen at around $8 \mathrm{meV}$ (labeled $\mathrm{A}$ in Fig. 6). Interestingly, in the phonon dispersion calculations of Souvatzis and Rudin [44] (using the large 192-atom supercell, Fig. 2 of Ref. [44]), while there is an instability (and imaginary frequencies) at the $X$-point as often reported in the literature, this band as it approaches the $W$-point, curves upwards becoming positive and exhibiting a maximum at about $8 \mathrm{meV}(2 \mathrm{THz})$ at the $W$-point. It is possible that this small region in which this band becomes positive at the $W$-point, is contributing the vDOS at this low energy.

Fig. 6 also shows the total and partial (atom-projected) vDOS of YSZ, which is displayed more clearly in Fig. 7. The greatest contributions to the vDOS for frequencies $<25 \mathrm{meV}$ are due to $\mathrm{Zr}$ and $\mathrm{Y}$ atoms, with the latter having a smaller contribution due to the fewer

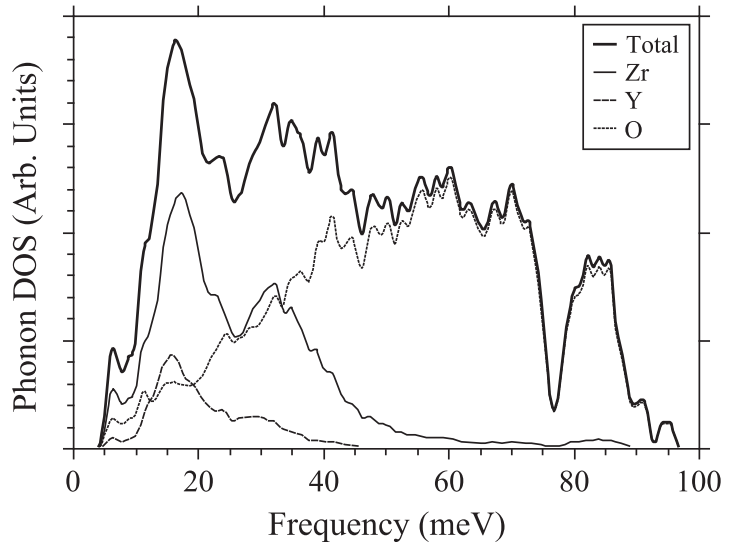

Fig. 7. Total and partial vDOS for YSZ (186 atom supercell) with $\sim 10 \mathrm{~mol} \% \mathrm{Y}_{2} \mathrm{O}_{3}$ Contributions from $\mathrm{Zr}$ and $\mathrm{Y}$ atom vDOS are mainly found at frequencies $<40 \mathrm{meV}$ and $\mathrm{O}$ vDOS dominate at higher frequencies ( $>40 \mathrm{meV})$.

$\mathrm{Y}$ atoms compared to $\mathrm{Zr}$ atoms. The higher contribution of $\mathrm{Zr}$ and $\mathrm{Y}$ to the vDOS at lower frequencies is because these atoms have larger masses than $\mathrm{O}$ and thus oscillate at lower frequencies than $\mathrm{O}$ atoms. Compared to $\mathrm{ZrO}_{2}$, the vDOS for YSZ are broadened, where the cation and O-related vibrations begin already at around $5 \mathrm{meV}$, and the O-related vibrations extend to just above $90 \mathrm{meV}$. A reason for this could be due to the $\mathrm{O}$-vacancies, in that on average the $\mathrm{Zr}-\mathrm{O}$ bonds for $\mathrm{Zr}$ atoms neighboring an $\mathrm{O}$-vacancy are shorter than the $\mathrm{Zr}-\mathrm{O}$ bonds for $\mathrm{Zr}$ atoms that are fully (8-fold) coordinated to oxygen atoms: Compare $2.228 \AA$ (fully coordinated), with $2.162 \AA$ for those $\mathrm{Zr}$ atoms with one O-vacancy as a nearest-neighbor. For $\mathrm{Zr}$ atoms with two O-vacancies as nearest-neighbor, the $\mathrm{Zr}-\mathrm{O}$ bond distance reduces slightly further, i.e. to $2.156 \AA$. These shorter, stiffer bonds are expected to give rise to higher frequencies. The phonon densityof-states of $c-\mathrm{ZrO}_{2}$, through their greater peaks and dips, in particular, the significant peak at around $14 \mathrm{meV}$, indicate that the phonon curves are more dispersive than those of YSZ. The vDOS of YSZ has more uniformly distributed phonon states. Comparing the total vDOS obtained in the present work with that of Lau and Dunlap [50] for the $40 \mathrm{~mol} \%$ yttria ( $\delta$-phase), and with that of Dalach et al. [32] (for 33 and 66 YSZ) it can be seen that the qualitative features are similar, namely, the vDOS are broadened in extent compared to $c-\mathrm{ZrO}_{2}$, and that the peak with a high vDOS at $\approx 14 \mathrm{meV}$ for $c-\mathrm{ZrO}_{2}$, is notably reduced in the YSZ system. In the result of Dalach et al. [32], the degree of this reduction is less significant than that of the present work, and than that of Lau and Dunlap [50].

The vibrational DOS at a given frequency are equivalent to the integration of phonon dispersion curves across the Brillouin zone at the corresponding energy transfer value; further, as mentioned above, peaks in vDOS at certain frequencies correspond to regions of the phonon dispersion curve that are flat (i.e. have low gradient). In this respect, consistent with the dispersion curves shown in Fig. 4 (where the energy of the $X_{1}$-point is notably higher for YSZ than pure $c-\mathrm{ZrO}_{2}$ ), the peak position of the vDOS (arrows "1" and "2" in Fig. 6) are at a higher energy compared to that of $\mathrm{ZrO}_{2}$ (labeled "B" in Fig. 6). This correlation, lends general support to the considered structural model. Furthermore, for YSZ, the vDOS shows a peak due to $\mathrm{Zr}$ at $17 \mathrm{meV}$ and due to $\mathrm{Y}$ at $16 \mathrm{meV}$. These features correspond well with the flat region in the measured dispersion curve (Fig. 4) at about $18 \mathrm{meV}$ near the $X$-point and $17 \mathrm{meV}$ about the $L$-point. Interestingly, the vDOS shows a shoulder at low energy i.e. 6-7 meV, (labeled "3") in Fig. 6 due mainly to $\mathrm{Zr}$-contributions. This raises the question of whether it can be related to the weak phonons measured in the $\Gamma-\Sigma-X$ direction (around 8-9 meV in Fig. 4). And further, whether this feature could be related to the longitudinal phonon mode that is imaginary for $c-\mathrm{ZrO}_{2}$, which on stabilization through yttria doping, 
shifts upwards and becomes positive. Clearly, further theoretical and experimental investigations are needed before any solid conclusions can be drawn.

\section{Conclusion}

The phonon dispersion curves for $8-9$ mol\% $\mathrm{Y}_{2} \mathrm{O}_{3}$ YSZ have been measured using inelastic neutron scattering measurements, and the vibrational density-of-states calculated using density-functional theory. The experimental results show good agreement with the earlier available work of Argyriou and Elcome [35], also obtained from inelastic neutron scattering measurements. Interestingly, the measurements find indication of weak, low energy phonons (8-9 meV) along the $\Gamma-\Sigma-X$ direction, previously not reported. For the calculated vDOS of cubic $\mathrm{ZrO}_{2}$, there is good agreement with earlier $a b$ initio calculations. For the calculated vDOS of YSZ, three prominent peaks at frequencies less than $25 \mathrm{meV}$ can be correlated with regions about the high symmetry points of the measured phonon dispersion curves. Two larger peaks relate to flat regions of the phonon dispersion curve at the Brillouin zone boundary $(X-$ and $L$-points). A smaller peak can be correlated to a flat region of phonon dispersion curve measured within the $X-\Sigma-\Gamma$ region of the Brillouin zone at around 8-9 meV as mentioned above, but requires further investigation. Consistent between the phonon measurements and calculated vDOS is that the cation-related phonons at the $X$-point are located at higher energies $(\approx 4-5 \mathrm{meV})$ for YSZ compared to the pure host material $c-\mathrm{ZrO}_{2}$, lending general qualitative support for the structural model.

\section{Acknowledgments}

Computing resources provided by the Australian National Computational Infrastructure (NCI) Facility and support from the Australian Research Council (ARC) are gratefully acknowledged.

\section{References}

[1] A.H. Heuer, F.F. Lange, M.V. Swain, A.G. Evans, J. Am. Ceram. Soc. 69 (3) (1986), i-iv.

[2] A.H. Heuer, L.W. Hobbs, Science and Technology of Zirconia, Advances in Ceramics, vol. 3, The American Ceramics Society, Inc, Columbia, OH, 1981.

[3] E.C. Subbarao, in: A.H. Heuer (Ed.), Advances in Ceramics, vol. 3, The American Ceramic Society, Inc, Columbia, OH, 1981.

[4] R.W. Cahn, P. Haasen, E.J. Kramer, Materials science and technology: a comprehensive treatment, in: B.R.T. Frost (Ed.), Nuclear Materials, VCH, New York, 1994.

[5] H.S. Hong, Y. Yongseung, K. Sublee, J. Alloy. Compd. 388 (2005) 279.

[6] J.S. Moya, M. Diaz, J.F. Bartolome, E. Roman, J.L. Sacedon, J. Izquierdo, Acta Mater. 48 (2000) 4749.

[7] R.H. French, S.J. Glass, F.S. Ohuchi, Y.-N. Xu, W.Y. Ching, Phys. Rev. B 49 (1994) 5133.
[8] R. Terki, G. Bertrand, H. Aourag, C. Coddet, Mater. Sci. Semicond. Process. 9 (2006) 1006.

[9] B. Králik, E.K. Chang, S.G. Louie, Phys. Rev. B 57 (1998) 7027.

[10] J.D. McCullough, K.N. Trueblood, Acta Crystallogr. 12 (1959) 507.

[11] P. Aldebert, J.P. Traverse, J. Am. Ceram. Soc. 68 (1985) 34.

[12] D.K. Smith, C.F. Cline, J. Am. Ceram. Soc. 45 (1962) 249.

[13] V.S. Stubican, J.R. Hellman, S.P. Ray, Mater. Sci. Monogr. 10 (1982) 257.

[14] S. Ostanin, A.J. Craven, D.W. McComb, D. Vlachos, A. Alavi, M.W. Finnis, A.T. Paxton, Phys. Rev. B 62 (2000) 14728.

[15] H.G. Scott, Acta Crystallogr. B: Struct. Crystallogr. Cryst. Chem. 33 (1977) 281.

[16] S. Ostanin, A.J. Craven, D.W. McComb, D. Vlachos, A. Alavi, A.T. Paxton, M.W. Finnis, Phys. Rev. B 65 (2002) 224109

[17] M.S. Khan, M.S. Islam, D.R. Bates, J. Mater. Chem. 8 (1998) 2299.

[18] X. Xia, R.J. Oldman, C.R.A. Catlow, J. Mater. Chem. 22 (2012) 8594.

[19] X.Q. Cao, R. Vaßen, D. Stoever, J. Eur. Ceram. Soc. 24 (2004) 1.

[20] S.D. Conradson, C.A. Degueldre, F.J. Espinosa-Faller, S.R. Foltyn, K.E. Sickafus, J.A. Valdez, P.M. Villella, Progr. Nucl. Energy 38 (2001) 221.

[21] D. Steele, B.E.F. Fender, J. Phys. C: Solid State Phys. 7 (1974) 1.

[22] J. Faber, M.H. Mueller, B.R. Cooper, Phys. Rev. B 17 (1978) 4884.

[23] M. Morinaga, J.B. Cohen, J. Faber, Acta Cryst. Sec. A 35 (1979) 789

[24] G. Morell, R.S. Katiyar, D. Torres, S.E. Paje, J. Llopis, J. Appl. Phys. 81 (1997) 2830

[25] J.P. Goff, W. Hayes, S. Hull, M.T. Hutchings, K.N. Clausen, Phys. Rev. B 59 (1999) 14202.

[26] D.N. Argyriou, M.M. Elcombe, A.C. Larson, J. Phys. Chem. Solids 57 (1996) 183.

[27] P. Li, I.-W. Chen, J.E. Penner-Hahn, J. Am. Ceram. Soc. 77 (1994) 118.

[28] A. Bogicevic, C. Wolverton, G.M. Crosbie, E.B. Stechel, Phys. Rev. B 64 (2001) 014106

[29] A. Bogicevic, C. Wolverton, Phys. Rev. B 67 (2003) 024106.

[30] D.M. Ramo, A.L. Shluger, J. Phys.: Conf. Ser. 117 (2008) 012022.

[31] A. Predith, G. Ceder, C. Wolverton, K. Persson, T. Mueller, Phys. Rev. B 77 (2008) 144104

[32] P. Dalach, D.E. Ellis, A. van de Walle, Phys. Rev. B 82 (2010) 144117.

[33] G.M. Stapper, M. Bernasconi, N. Nicoloso, M. Parrinello, Phys. Rev. B 59 (1999) 797.

[34] W. Liu, C.H. Perry, A.A. Feinberg, R. Currat, Phys. Rev. B 36 (1987) 9212.

[35] D.N. Argyriou, M.M. Elcombe, J. Phys. Chem. Solids 57 (1996) 343.

[36] A.P. Mirgorodsky, M.B. Smirnov, Phys. Rev. B 55 (1997) 18.

[37] A.P. Mirgorodsky, M.B. Smirnov, P.E. Quintard, J. Phys. Chem. Sol. 60 (1999) 985.

[38] A.P. Mirgorodsky, M.B. Smirnov, T. Merle-Mejean, P.E. Quintard, J. Mater. Sci. 34 (1999) 4845.

[39] K. Parlinski, Z.Q. Li, Y. Kawazoe, Phys. Rev. Lett. 78 (1997) 4063.

[40] F. Detraux, Ph. Ghosez, X. Gonze, Phys, Rev, Lett. 81 (1998) 3297.

[41] S. Fabris, A.T. Paxton, M.W. Finnis, Phys. Rev. B 63 (2001) 094101.

[42] M. Sternik, K. Parlinski, J. Chem. Phys. 122 (2005) 064707.

[43] A.T. Kuwabara, T. Tohei, T. Yamamoto, I. Tanaka, Phys. Rev. B 71 (2005) 064301.

[44] P. Souvatzis, S.P. Rudin, Phys. Rev. B 78 (2008) 184304.

[45] G. Fadda, G. Zanzotto, L. Colombo, Phys. Rev. B 82 (2010) 064106.

[46] G. Fadda, G. Zanzotto, L. Colombo, Phys. Rev. B 82 (2010) 064105.

[47] T. Tojo, H. Kawaji, T. Atake, Solid State Ionics 118 (1999) 349.

[48] P.K. Shelling, S.R. Phillpot, D. Wolf, J. Am. Ceram. Soc. 84 (2001) 1609

[49] S. Ostanin, E. Salamatov, JETP Lett. 74 (2001) 552.

[50] K.C. Lau, B.I. Dunlap, J. Phys.: Condens. Matt. 21 (2009) 145402.

[51] G.P. Cousland, X.Y. Cui, A.E. Smith, C.M. Stampfl, L. Wong, M.Tayebjee, D. Yu, G. Triani, A.P.J. Stampfl, unpublished.

[52] S.A. Danilkin, G. Horton, R. Moore, G. Braoudakis, M. Hagen, J. Neutron Res. 15 (2007) 55.

[53] G. Shirane, S.M. Shapiro, J.M. Tranquada, Neutron Scattering with a Triple-Axis Spectrometer, Cambridge University Press, 2002.

[54] S.A. Danilkin, M. Yethiraj, Neutron News 20 (2009) 37.

[55] B. Delley, J. Chem. Phys. 113 (2000) 7756.

[56] J.P. Perdew, K. Burke, M. Ernzerhof, Phys. Rev. Lett. 77 (1996) 3865.

[57] G.P. Cousland, X.Y. Cui, A.E. Smith, A.P.J. Stampfl, C. Stampfl, unpublished.

[58] E.V. Stefanovich, A.L. Shluger, C.R.A. Catlow, Phys. Rev. B 49 (1994) 11560. 